\title{
Controlled lateral and perpendicular motion of atoms on metal surfaces
}

\author{
A. Buldum and S. Ciraci \\ Department of Physics, Bilkent University, Bilkent 06533, Ankara, Turkey
}

(Received 14 November 1995)

\begin{abstract}
We present the theoretical study of the controlled lateral and perpendicular motion of $\mathrm{Xe}$ on the $\mathrm{Pt}(111)$ surface. The lateral translation of Xe is manipulated by a tungsten tip of a scanning tunneling microscope. Using molecular statics and dynamics the energetics and different modes of atom translation are revealed. In the controlled and reversible transfer of Xe between two flat $\mathrm{Pt}(111)$ surfaces, effective charge on Xe, and the dipole moment of the Xe-Pt bond, are calculated as functions of the Xe-surface separation. The contributions of various mechanisms to the transfer rate of Xe are investigated by using the calculated quantum states of Xe under the applied bias voltage. These are tunneling and ballistic transfer, dipole excitation and excitation due to resonant tunneling of electrons, and electron wind force. We found that a single power law for the transfer rate does not exist in the whole range of applied pulse voltage. At high pulse voltage the transfer rate is dominated by the inelastic electron tunneling. At low pulse voltage the rate due to thermally assisted tunneling and ballistic transfer becomes important. [S0163-1829(96)03527-8]
\end{abstract}

\section{INTRODUCTION}

Recent developments made in scanning tunneling microscopy (STM), such as translation and relocation, ${ }^{1}$ controlled diffusion, ${ }^{2}$ desorption and dissociation of atoms at surfaces, 3,4 and reversible transfer of atoms between tip and sample surface, ${ }^{5}$ have demonstrated that the manipulation and modification of matter on the atomic scale is now possible. While surface physics has made tremendous progress related to static interactions of various surfaces with adsorbed atoms or molecules for two decades, the controlled motion of adsorbed species has opened a new field of research with interesting physics and perhaps with future potential applications. ${ }^{6}$ The effects of tip-sample interaction on the electronic and atomic structure at close proximity to the tip have been the subject of earlier studies. ${ }^{7,8}$ Here, the tipadatom-surface interaction modifies the potential energy and may introduce new local minima for the adsorbate on the Born-Oppenheimer surface for a given position of the tip. Usually these minima follow the motion of the tip. External agents, such as external electric field, photons, and electrons can influence the controlled dynamics of atoms. In the course of the controlled motion the adatom can be also transferred from sample to tip. The idea that an atom can be transferred between the tip and sample surface is due to Gomer. ${ }^{9}$ The potential energy of the adsorbate between tip and sample surface (or between two electrodes) forms a double-well structure; the energy barrier between wells is crucial for the atom transfer. The atom transfer is simply the crossing of the potential barrier between two wells. This barrier is modified by changing the tip-sample separation and/or by applying a voltage pulse. Earlier, the variation of the double-well potential corresponding to open- and closed-shell atoms between two metal surfaces has been investigated from first principles. $^{10}$

The mechanisms responsible for the controlled motion of atoms by STM have been treated recently. Some studies have investigated the lateral translation of inert gas atoms adsorbed on the metal surfaces by using $a b$ initio $^{11}$ and em- pirical potentials. ${ }^{12,13}$ The dynamics and different modes of lateral motion on the metal surface are now well understood. The mechanism of transfer between two electrodes is known for strongly bound atoms, such as a $\mathrm{Si}$ atom between a $\mathrm{Si}$ surface and a tungsten $\mathrm{tip}^{3}$ or a $\mathrm{Au}$ atom between gold electrodes. ${ }^{4}$ However, the physical phenomena involved in the reversible transfer of Xe between the Ni surface and $\mathrm{W}$ tip (so-called atom switch) have not been clarified yet. Eigler, Lutz, and Rudge ${ }^{5}$ found power-law dependence of the transfer rate on the pulse voltage according to $V^{4.9}$ and proposed heating assisted electromigration as the mechanism consistent with their observations. However, the power-law dependence has been disputed and different mechanisms are put forward to explain the reversible transfer in recent theoretical studies. ${ }^{14-18}$ The key issues to be resolved are now the verification of the power-law dependence and the clarification of the role of different mechanisms in the transfer rate for a different range of applied pulse voltage.

It is clear that the form of the double-well potential, especially the height and width of the energy barrier, are crucial for the transfer of Xe. The energy barrier, in turn, depends on the form of the empirical potential. Moreover, the variation of the height of the barrier under the pulse voltage is strongly dependent on the excess charge of Xe. Similarly, the variation of the dipole moment of the Xe-Pt bond is important for the vibrational excitation of the adatom.

This work presents the theoretical study of the controlled lateral and perpendicular motion of a Xe atom on the $\mathrm{Pt}(111)$ surface, for which the interaction between $\mathrm{Xe}$ and $\mathrm{Pt}$ atoms can be accurately represented by an empirical potential due to Barker and Rettner. ${ }^{19}$ This potential is consistent with a wide range of dynamical and equilibrium data and yields that the equilibrium position of the single $\mathrm{Xe}$ atom lies directly above a surface platinum atom. Recently, a self-consistentfield (SCF) cluster calculation ${ }^{20}$ indicated also the top site as the adsorption position of $\mathrm{Xe}$ on the $\mathrm{Pt}(111)$ surface. For lateral motion we used a W(111) tip and examined the potential energy surface, which is essential for the dynamics of adatom. Further, in regard to earlier experiments and the 
simulations related with the lateral translation of $\mathrm{Xe}$ on the $\mathrm{Ni}(110)$ surface, the present results clarify the role of surface structure, adsorption site, and material parameters.

The study of the perpendicular motion of Xe is carried out between two flat $\mathrm{Pt}(111)$ slabs. This way we simplify the problem by eliminating some unknown factors existing in the transfer process. For example, we do not deal with the uncertainties due to the effect of the adsorption site and tip structure. The objective of this paper is to provide appropriate treatments for the effective charge of Xe and the dipole moment of the Xe-Pt bond, and to reveal the contributions of various mechanism in the transfer rate.

\section{LATERAL TRANSLATION OF Xe ON Pt(111)}

The interaction energy between xenon and the $\operatorname{Pt}(111)$ surface consists of (i) short-range and attractive interaction energy due to the charge rearrangements in the chemical bond, (ii) short-range repulsive energy, (iii) long-range and attractive van der Walls energy. It is usually argued that the charge rearrangement upon physisorption of closed-shell atoms, such as Xe on metal surfaces, is negligible, and hence does not induce any short-range attractive interaction. On the contrary to this argument, the local density treatment of the interaction between closed-shell atoms and jellium metal surfaces by Lang ${ }^{21}$ provided a good account of experimental data on atomic binding energy, dipole moment, and corelevel binding energy shift. By examining work-function measurements of rare-gas atoms adsorbed on metal surfaces Ishi and Viswanathan ${ }^{22}$ concluded that the chemical binding effects are essential in bonding. Recently, Baratoff, Ciraci, and Stoll ${ }^{11}$ carried out a self-consistent field pseudopotential calculation with the local density approximation for the binding energy of $\mathrm{Xe}$ on the $\mathrm{Al}(111)$ surface. The calculated binding energy was $130 \mathrm{meV}$, which is compared to the experimental binding energy of $200 \mathrm{meV}$. This indicates that even for the adsorbed closed-shell atoms the short-range interaction near the equilibrium distance dominates the weak long-range interaction. The free parameter linear combination of atomic orbitals (LCAO) calculation by Pérez et al. ${ }^{23}$ also confirms this conclusion.

In the present calculations of lateral motion the Pt surface is represented by $42 \mathrm{Pt}(111)$ atomic layers comprising $14112 \mathrm{Pt}$ atoms. The tip is constructed by $2024 \mathrm{~W}$ atoms in pyramidal geometry generated from $22 \mathrm{~W}(111)$ layers. The apex of the tip has a single atom; the second layer has three atoms. The coordinates of the tip (apex atom) and those of Xe relative to a point on the sample surface are labeled by $(x, y, z)$ and $(\xi, \kappa, \zeta)$, respectively. The interaction potential between the Xe atom (at $\mathbf{R}_{a}$ ) and any atom of the W tip (at $\left.\mathbf{R}_{l}\right)$ is expressed in terms of a Lennard-Jones pair potential having the well-known form $\epsilon\left[\left(r_{0} /\left|\mathbf{R}_{a}-\mathbf{R}_{l}\right|\right)^{12}-2\left(r_{0} / \mid \mathbf{R}_{a}\right.\right.$ $\left.\left.-\mathbf{R}_{l} \mid\right)^{6}\right]$. The parameters of this potential are determined ${ }^{13}$ from the experimental data to be $\epsilon=0.339 \mathrm{eV}$, and $r_{0}=3.62 \AA$. The many-body effects are taken into account by scaling $\epsilon$ and $r_{0}$ values. Note that the Lennard-Jones pair potential function is only a crude approximation for the $\mathrm{Xe}-\mathrm{W}$ interaction; it could have been improved by using additional terms. This, however, requires additional experimental data to fit, which are not available yet. The atomic arrangement of the tip-adatom-surface system and the
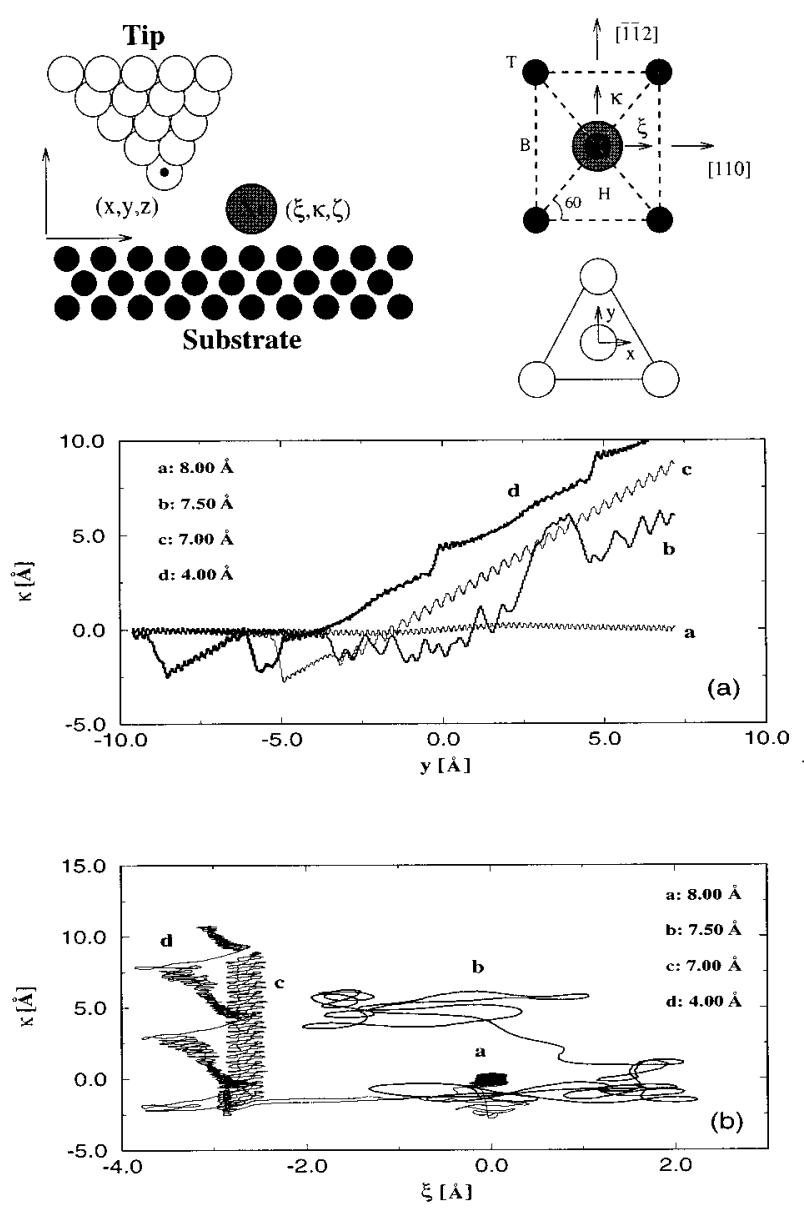

FIG. 1. Lateral translation of Xe on the $\mathrm{Pt}(111)$ surface induced by the $\mathrm{W}$ tip moving along the $y[\overline{11} 2]$ direction. $a, b, c$, and $d$ correspond to different heights $z$ of the tip. Trajectories of Xe in the $(\kappa, y)$ and $(\kappa, \xi)$ planes are shown in the top and bottom panels, respectively. The direction and orientation of the tip relative to the $\operatorname{Pt}(111)$ unit cell are shown schematically.

orientation of the tip are schematically described in Fig. 1.

The empirical potential introduced by Barker and Rettner ${ }^{19}$ expresses the interaction between a single Xe atom and the $\operatorname{Pt}(111)$ surface in terms of the sum of nonspherical, pairwise additive potentials and of an additional term, which describes the interaction of Xe with the delocalized conduction electrons of the sample surface. The nine parameters in the potential function were fitted to a wide range of available experimental data. Details of this potential can be obtained in Ref. 19.

The electrodes [i.e., the $\mathrm{W}$ tip and the $\mathrm{Pt}(111)$ substrate] are taken rigid. As a result, the position of atoms in these electrodes are fixed at their bulk equilibrium positions and hence part of the interatomic interactions (such as Pt-Pt, Pt-W, and W-W) are not taken into account in the total potential function. The assumption of rigid electrodes is valid if the height $z$ of the tip is large. For relatively smaller tipsample separations the modification of the atomic structure at close proximity to the tip (such as elastic or plastic deformation, wetting, and surface melting) has to be taken into account, however. ${ }^{13}$

The potential energy surface of $\mathrm{Xe}$ is calculated at each 
TABLE I. Energetics of Xe on the Pt(111) surface. $T, H, B$ denote for top, hollow, and bridge sites, respectively.

\begin{tabular}{lccc}
\hline \hline & Binding energy $E_{b}(\mathrm{meV})$ & Barrier energy $Q(\mathrm{meV})$ \\
\hline & & & 32 \\
$H$ site & 222 & $T \rightarrow H \rightarrow \mathrm{T}$ & 26 \\
$T$ site & 254 & $T \rightarrow B \rightarrow \mathrm{T}$ & \\
$B$ site & 228 & & \\
\hline \hline
\end{tabular}

grid point $(\xi, \kappa)$ on the $\operatorname{Pt}(111)$ surface by varying its height $\zeta$. Then, the potential energy surface is obtained by plotting the minimum values of the calculated energy at each grid point. The highest binding energy (or lowest potential energy) is $E_{b}=254 \mathrm{meV}$ and occurs at the top site. This is in contrast to many adsorbate-substrate systems [such as $\mathrm{Xe}$ on the $\mathrm{Al}(100)$ surface] in which the hollow site occurs as the equilibrium binding site. The change of the adsorption site on the Pt surface is attributed to the reduction of $s+p$ electron density due to the $d$ states occupied near the Fermi level. ${ }^{23}$ The binding energy at the symmetry points of the unit cell and the energy barriers for different directions of motion are shown in Table I.

The potential energy surface is strongly modified by a W tip approaching the physisorbed Xe atom. The minimum of the potential energy, which is displaced towards the tip, usually follows the motion of the tip, if the tip-sample distance is properly varied in the course of translation. The carriage of $\mathrm{Xe}$ on the $\mathrm{Pt}(111)$ surface as a function of the height of the tip $z$ is studied by performing molecular dynamics calculations. The results for the tip moving along the $y$ direction from a distance towards Xe at different heights $z$ are summarized in Fig. 1. The tip moves from a distance towards Xe for different values of $z$. For $z=8 \AA$, the Xe atom remains practically unaffected. At $z=7.5 \AA$, the interaction between the tip and Xe becomes significant; first it is attracted by the motion of the tip, then it follows the tip for a very short distance, but then it becomes unaffected by the tip. For $5 \AA<z<7 \AA$, Xe is attached to the tip and is then carried without showing any periodicity due to the surface corrugation. For this range of $z$ the interaction sets in already at $\kappa-y=5 \AA$ since the second layer of tip atoms is closer to $\mathrm{Xe}$; the Xe atom escapes $\sim 2.7 \AA$ sideways to the adjacent unit cell and at the same time is attached to the side face of the W tip. Only for a smaller height of the tip $(z<5 \AA)$ is the corrugation of the $\mathrm{Pt}(111)$ surface reflected to the trajectory of Xe. In this case, Xe escapes again sideways by $\sim 2.7 \AA$ to the adjacent top site and pushed by the tip moving along the [112] direction. The jumps of the curve $d$ in the upper panel correspond to the $\mathrm{Xe}$ atom from one $T$ site to the next one along the $[\overline{11} 2]$ direction. In the bottom panel of Fig. 1 we show the $(\kappa, \xi)$ trajectories of Xe corresponding to the same set of $z$ in panel (a). The Xe atom carried by the tip at $z=7 \AA$ traces a straight route along the $\kappa$ axis with $\xi$ fixed at $\sim 2.7 \AA$. For $z=4 \AA$, the zigzag trajectory is produced by Xe, which is pushed from one $T$ site to the next one along the [ $\overline{112}$ ] direction by avoiding the $H$ site. This behavior of pushing mode is rather different from that found in the controlled motion of $\mathrm{Xe}$ on the $\mathrm{Ni}(110)$ surface. ${ }^{12,13}$

The translation of Xe along the [110] direction (or $x$ di- rection) is similar to the one along the [ $\overline{112}$ ] direction described above. The energy barrier in the lateral motion of Xe on the $\mathrm{Pt}(111)$ surface is smaller than that on the $\mathrm{Ni}(110)$ surface. This is because the $\operatorname{Pt}(111)$ surface is a closedpacked plane and the surface charge density has relatively smaller corrugation. Due to this fact the lateral translation of Xe on the surface exhibits the periodicity of the substrate only in a certain range of $z$. The trajectories presented in Fig. 1 are closely related to the two-dimensional stick-slip motion in the science of friction. The energy damping from this motion is of current interest.

\section{REVERSIBLE TRANSFER OF Xe BETWEEN FLAT Pt(111) ELECTRODES}

The atom transfer between two electrodes has been realized for weakly as well as strongly bound adsorbates. Mamin, Guethner, and Rugar ${ }^{4}$ achieved the transfer of gold atom from a negatively biased gold tip to a substrate. They argued that negative Au-ion formation and subsequent field evaporation is responsible for the transfer. Lyo and Avouris, ${ }^{3}$ who realized reversible atom transfer between the tip and $\mathrm{Si}$ substrate, have suggested that the transfer takes place by ionization (positive Si-ion formation) followed by field evaporation. The controlled and reversible transfer of the weakly bound $\mathrm{Xe}$ atom between the $\mathrm{Ni}(110)$ surface and a $\mathrm{W}$ tip, so-called atom switch, ${ }^{5}$ displayed characteristics that are quite different from the two former cases: ${ }^{3,4}$ For example, (i) $\mathrm{Xe}$ is always transferred towards the positively biased electrode. (ii) At small tip-sample distance (corresponding to the junction resistance $R<700 \mathrm{k} \Omega$ ) the Xe atom moves spontaneously to the tip without the need to apply a positive pulse voltage. (iii) At larger separation $(R>1.5 \mathrm{M} \Omega)$, however, Xe either hops on the Ni surface or escapes from the junction entirely. (iv) Depending upon the location of the Xe atom [at kink sites, on the bare $\mathrm{Ni}(110)$ terrace, on top of a single $\mathrm{Ni}$ adatom on the $\mathrm{Ni}(110)$ surface] the conductance ratio ranges from unity to 7 . (v) There is a characteristic transfer rate for a fixed sample distance. For example, it is claimed ${ }^{5}$ that for a 906-k $\Omega$ junction the transfer rate has a power-law dependence on the pulse voltage, according to $\tau^{-1} \approx V_{P}^{4.9 \pm 0.2}$. Gao, Persson, and Lundqvist ${ }^{14}$ examined various physical mechanisms responsible for the reversible transfer of Xe between the $\mathrm{Ni}(110)$ surface and a $\mathrm{W}$ tip, and proposed vibrational heating by inelastic electron tunneling to explain the powerlaw dependence reported by Eigler, Lutz, and Rudge. ${ }^{5}$ They also concluded that the multiple (incoherent) vibrational excitation via inelastic electron tunneling plays an important role. Later, Salam, Persson, and Palmer ${ }^{17}$ argued that for other systems, such as $\mathrm{Na}$ on $\mathrm{Cu}$, the coherent multiple excitation of the adsorbate-substrate bond caused by inelastic 
tunneling of a single electron (or hole) via negative (or positive) ion resonance dominates the vibrational heating (incoherent excitation). They found the vibrational heating mechanism to dominate over the coherent mechanism in the case of the atom switch. ${ }^{5}$ On the other side, Saénz and Garcia $^{15}$ proposed that the experimentally observed atom transfer process 5 complies with a thermally assisted singleatom tunneling. They argued that the transfer rate cannot follow a power-law dependence with the applied pulse at small voltages. Moreover, they were able to reproduce the observed dependence of the transfer rate on the applied pulse at the high voltage region only by using unrealistically large and constant (and negative) effective charge $\left(q_{\text {eff }}=0.3 e\right)$ on the physisorbed $\mathrm{Xe}$ atom.

\section{A. Variation of potential energy of Xe}

The potential energy of Xe between two flat $\mathrm{Pt}(111)$ surfaces separated by $z$ is $U\left(\xi, \kappa, \zeta, z ; V_{P}\right)$ and has double minima for $z>2 \zeta_{0}$ [ $\zeta_{0}$ being the equilibrium distance of Xe adsorbed on the $\operatorname{Pt}(111)$ surface]. Two wells (each being closer to one of the Pt surfaces) determine the equilibrium distance and energy of Xe between two electrodes but adsorbed only to one of them. As $z$ decreases the energy barrier $Q(z)$ is lowered and eventually collapses, leading to a single well. Since we are interested in the motion along $\zeta$, we adopt a one-dimensional model and consider only the variation of $U$ along the [111] direction. Implementation of the other directions would bring new eigenstates, which do not play a crucial role in the transfer rate. For the system used in the present study $U(\zeta, z)$ is symmetric in the absence of applied bias voltage.

We generate the potential (energy) function $U(\zeta, z)$ of Xe between two parallel and flat $\mathrm{Pt}(111)$ electrodes by using Barker and Rettner ${ }^{19}$ potential described in Sec. II. At relatively small $z$, this potential gives us a shallow well at the center in addition to two deep wells. It occurs perhaps due to the limitation of the interpolation between the short-range and the long-range part of the potential function. If this were the real situation, the resonant tunneling of atoms would occur across the wells in appropriate conditions. This artifact of the potential is corrected by improving the interpolation between two ranges of the interaction. In Fig. 2 we illustrate the potential energy curves along the [111] direction (or the $\zeta$ direction) for different separation $z$, and variation of $Q$ with $z$. The symmetry of $U(\zeta, z)$ is broken by applying a pulse voltage if the adsorbed Xe is charged and/or the Xe-Pt bond is polarized. In this case one well is lowered relative to the other, and also the energy barrier is decreased. This way not only the directionality but also the control of Xe transfer becomes possible.

Apparently, the effective charge of the adsorbed Xe as well as the charge distribution of the Xe-Pt bond are two features that are critical ingredients of the atom transfer. The physisorption of Xe on simple metals as well as on transition metals results in the reduction of the work function ${ }^{22,24} \Phi$. This is $\sim 0.96 \mathrm{eV}$ for the density of $\sim 6 \times 10^{14} \mathrm{Xe}$ atoms per $\mathrm{cm}^{2}$ on a platinum ${ }^{24}$ surface. This implies that the Xe-Pt bond induces a dipole moment that is in the reverse direction to that of the bare surface. The dipole moment leading to the work function lowering $\Delta \Phi \simeq-0.96 \mathrm{eV}$ is determined by
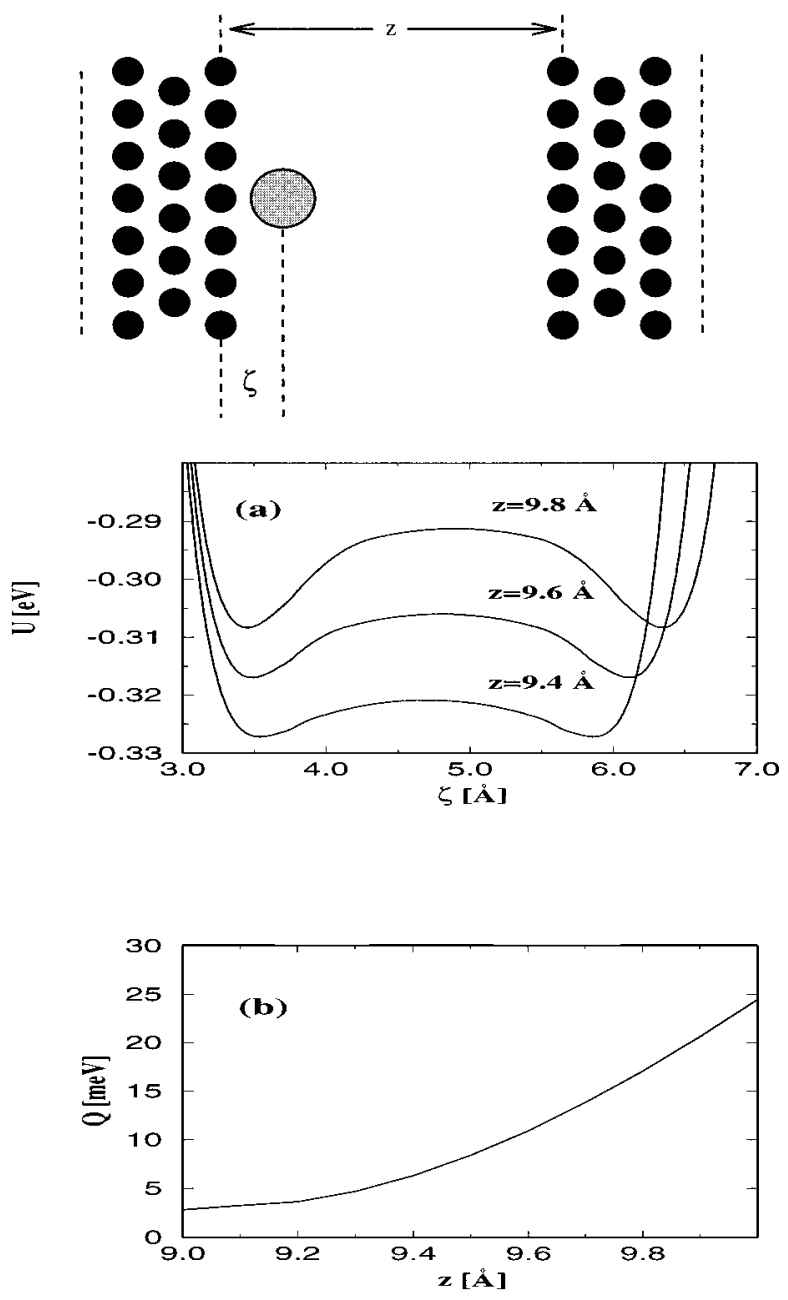

FIG. 2. (a) Variation of the potential energy of Xe, $U(\zeta, z)$, with the separation of the $\operatorname{Pt}(111)$ electrodes $z$. (b) Variation of the barrier energy $Q(z)$ with separation. The bias voltage $V_{P}=0$. The arrangement of electrodes and the relevant coordinates are described schematically.

using Topping's formula ${ }^{25}$ to be $0.65 \mathrm{D}$. The character and charge distribution of the bond between an adsorbed inert gas atom and the metal surface have been the subject of several studies. Unfortunately, theoretical results reported to date are not conclusive. A recent local density approximation calculation by Müller ${ }^{20}$ suggests that the Xe $5 p$ orbital makes bonding and antibonding combinations with occupied metal states. On the other hand, as a result of the combination of the Xe $5 p$ state and partially filled Pt $5 d$ state, the charge $\Delta q=0.085$ electrons is transferred from Xe to the metal. This provides the binding by lowering the Xe $5 p$ level by $\Delta q\left(E_{F}-\epsilon_{\mathrm{Xe5p}}\right)$. Clearly, the local bonding and the charge transfer are usually emphasized in the cluster calculations. ${ }^{20}$ Based on the atom-on-jellium calculations, Eigler et $a l .{ }^{26}$ proposed that the empty Xe $6 s$ level, which lies above the Fermi level, is broadened upon the adsorption of Xe on the metal surface and becomes partially occupied. Hence, the finite local density of states at the Fermi level, $\rho\left(r, E_{F}\right)$ renders Xe visible in the STM. The existence of this so-called $s$-resonance model for Xe adsorbed on the metal surfaces was suggested several years ago. ${ }^{27}$ Clearly, the par- 
tial occupation of the Xe $6 s$ resonance by the metal electrons indicates the charge transfer from the metal to the adsorbed Xe. Such a broadening of the empty adsorbate level does not occur in a metal cluster consisting of a few metal atoms as in the model of Müller. ${ }^{20}$

The charge transfer of Xe is in agreement with the findings of the SCF pseudopotential calculation. ${ }^{11}$ The freeparameter LCAO calculation by Pérez et al. ${ }^{23}$ also finds that charge is transferred from the $\mathrm{Al}(100)$ surface to the adsorbed Xe to yield $q_{\text {eff }} \sim 0.1$ electron. Experimentally, Wandelt and Gumhalter ${ }^{28}$ found direct support from the ultraviolet photoemission spectroscopy of the valence band of the $\mathrm{Xe}$-covered Pd surface that the $\mathrm{Xe} 6 s$ resonance is partially occupied and the physisorbed $\mathrm{Xe}$ becomes negatively charged.

The partial filling of the $\mathrm{Xe} 6 s$ resonance leading to a charge transfer to the adsorbed Xe is estimated ${ }^{29}$ by using the chemisorption theory ${ }^{30}$ based on Anderson's Hamiltonian ${ }^{31}$ where the states of the metal-adatom system $|\phi\rangle$ are expanded in terms of the adatom $|a\rangle$ and metal $|k\rangle$ states. Then the density of states localized in a particular adatom state $|a\rangle$ is $\rho_{a}(\epsilon)=\Sigma_{\phi}|\langle\phi \mid a\rangle|^{2} \delta\left(\epsilon-\epsilon_{\phi}\right)$. This is formulated ${ }^{30}$ as

$$
\rho_{a}(\epsilon)=\frac{\Gamma}{\pi}\left[\left(\epsilon-\epsilon_{a}-\Lambda\right)^{2}+\Gamma^{2}\right]^{-1},
$$

which is approximately centered at $\epsilon=\epsilon_{a}+\Lambda$ and has half width at half maximum $\Gamma$. It is seen that the empty state of a free Xe atom is broadened and displays a Lorentzian-like distribution upon adsorption on a metal surface. The tail of this resonance dips in the Fermi level and becomes partially occupied. That is $n=1 / 2-\pi^{-1} \arctan (\epsilon / \Gamma)$. In the present case $\Gamma$ is expressed as

$$
\Gamma \approx \pi \mid\left\langle\psi_{\mathrm{Xe} 6 s} \mid \psi_{\mathrm{Pt} 6 s}\right\rangle \frac{\epsilon_{\mathrm{Xe} 6 s}+\left.\epsilon_{\mathrm{Pt} 6 s}\right|^{2}}{2},
$$

where the local overlap $\left\langle\psi_{\mathrm{Xe} 6 s} \mid \psi_{\mathrm{Pt} 6 s}\right\rangle$ is calculated numerically by using the Herman-Skillman ${ }^{32}$ wave function and is found $\sim 0.44$ for the equilibrium adatom-substrate distance, $\zeta=\zeta_{0}$. Since the spin-polarized electron spectroscopy ${ }^{33}$ sets the position of the $\epsilon_{\mathrm{Xe} 6 s}$ level, $\epsilon_{a}+\Lambda, 3.91 \mathrm{eV}$ above the Fermi level, the partial filling of the $\mathrm{Xe} 6 s$ resonance is found to be 0.08 per spin at the equilibrium distance $\zeta_{0}$ of the adsorbed Xe. Note that the occupation of the Xe $6 s$ resonance varies with the distance $\zeta$ between $\mathrm{Xe}$ and the $\mathrm{Pt}(111)$ surface in the course of the transfer. The effective charge of Xe between two $\operatorname{Pt}(111)$ electrodes, $q_{\text {eff }}(\zeta, z)$ is estimated by adding the excess charge on Xe originating from each electrode, $\Delta q(\zeta)$ and $\Delta q(z-\zeta)$. Of course, the additivity of $\Delta q$ associated with each electrode is justifiable only for relatively large $z$. At small $z$, the interelectrode interaction has to be taken into account. Figure 3 illustrates the variation of the effective charge $q_{\text {eff }}(\zeta, z)$ for a given $z$. Finally, because of $q_{\text {eff }}$ the potential energy $U(\zeta, z)$ is modified under the applied pulse voltage $V_{P}$. That is

$$
\Delta U\left(\zeta, z ; V_{P}\right)=-q_{\mathrm{eff}}(\zeta, z) \frac{\zeta-d_{i}}{z-z_{i}} V_{P},
$$

where $d_{i}$ is the image plane position ${ }^{34}$ for the first atomic plane of the electrode and $z_{i}=2 d_{i}$. Figure 4(a) illustrates the

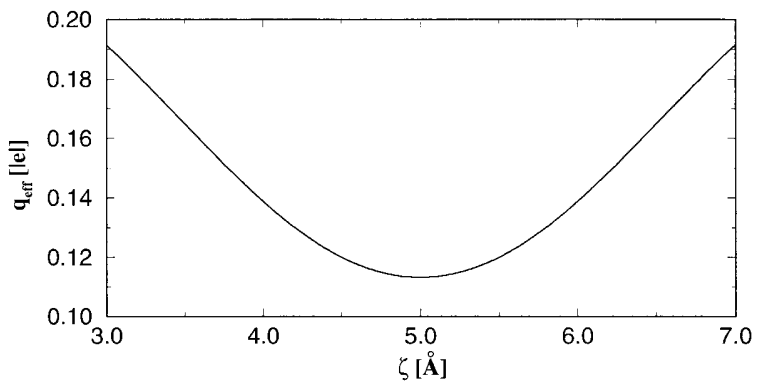

FIG. 3. Variation of the effective charge $q_{\text {eff }}(\zeta, z)$ on Xe between two $\operatorname{Pt}(111)$ electrodes separated by $z=10.0 \AA$.

variation of the potential energy with different applied pulse voltages. Note that for a given $z$ and $V_{P}, \Delta U$ decreases linearly and reduces the barrier only when $q_{\text {eff }}$ is constant. However, the variation of the barrier with $\zeta$ is not obvious if the value of the $q_{\mathrm{eff}}$ varies with $\zeta$. For example, for $\Delta q(\zeta) \sim e^{-\beta \zeta}, Q$ increases for $\beta>0.88$, but decreases for $\beta \leqslant 0.88$ for $z=10 \AA$. In Fig. 4(b), the variation of $Q\left(z ; V_{P}\right)$ is shown for calculated $q_{\mathrm{eff}}(\zeta, z)$, as well as for constant $q_{\text {eff }}$. Note that the barrier lowering with $V_{P}$ becomes dramatic if $q_{\text {eff }}$ is taken constant. In view of the present results, earlier conclusions based on the constant $q_{\text {eff }}$ are seriously questioned.

The change of $U$ with $V_{P}$, and hence the directionality of atom transfer, can also be obtained from the adsorptioninduced dipole $^{23}$ as done by Walkup, Newns, and Avouris. ${ }^{16}$
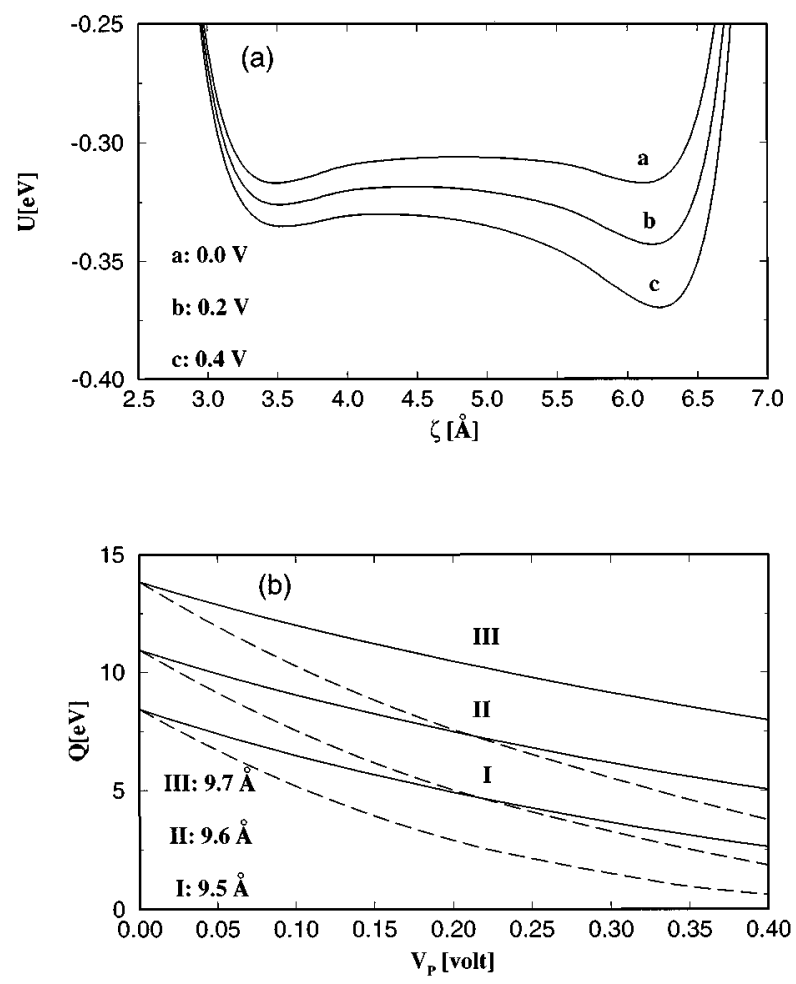

FIG. 4. (a) Variation of the potential energy $U\left(\zeta, z ; V_{P}\right)$ of Xe between two $\operatorname{Pt}(111)$ electrodes with different applied bias voltage $V_{P}$ for $z=9.6 \AA$. (b) Variation of the energy barrier $Q\left(z ; V_{P}\right)$ with applied bias voltage $V_{P}$ for three different electrode separations $z$ (I,II,III). The dashed curves correspond to $Q\left(z ; V_{P}\right)$ where $q_{\text {eff }}(\zeta, z)$ is constant and equals 0.16 electron. 
The dipole takes on opposite signs for adsorption on the sample and tips, and it provides the observed directionality of the Xe motion. The calculation of dipole moment, however, requires that the distribution of charge at the Xeadsorbed semi-infinite $\mathrm{Pt}$ surface is obtained selfconsistently. Yet such a calculation is not available. In our approach we calculate $\Delta U\left(\zeta, z ; V_{P}\right)$ by using $q_{\text {eff }}$ which is obtained unambiguously from the chemisorption model. ${ }^{30,31}$ A similar model in a much simpler and parametrized form was used by Saénz and Garcia. ${ }^{15}$ Both approaches used in calculating $\Delta U$ (i.e., one due to Walkup, Newns, and Avouris ${ }^{16}$ and one used here) are acceptable and interrelated. We note that the excess charge on Xe due to the $6 s$ resonance (which is also crucial for the STM image of Xe) does not contradict the lowering of the work function as a result of Xe adsorption. The dipole moment leading to the workfunction lowering is obtained from the integration of the charge near the surface, and hence even a slight polarization of charge on Xe may induce significant dipole moment. The dipole moment $\mu$ of the Xe-Pt bond that lowers the work function is made from three major components, ${ }^{28}$ namely,

$$
\mu=\mu_{s}+\mu_{d}+\mu_{q} .
$$

These are, respectively, static polarization, dynamic polarization, and charge transfer components. Here, $\mu_{s}+\mu_{d}$ and $\mu_{q}$ are competing contributions. While the work function is reduced by $\mu_{s}+\mu_{d}, \mu_{q}$, which has the opposite sign of $\mu_{s}+\mu_{d}$, raises $\Phi$. The origin and the character of these components are extensively discussed and contrasted with reverse face specificity of $\Phi$ and adsorption energy by Wandelt and Gumhalter. ${ }^{28}$ They even pointed out experimental work on the Cs surface, where the adsorption of Xe having $\mu_{q}>\mu_{s}+\mu_{d}$ leads to an increase of $\Phi$.

As in $q_{\text {eff }}(\zeta, z)$, the variation of $\mu$ with the distance from the metal surface is also needed in the study of atom transfer. $\mu_{q}$ varies as $q_{\text {eff }}$ does, since it is equal to $2 \bar{d}_{i} q_{\text {eff }}$. Here $\bar{d}_{i}=\zeta-d_{i}$. As for $\mu_{s}$ we assume the form $\mu_{s}=C S \bar{d}_{i}$ in terms of the overlap $S$ between Pt $5 d_{20}$ and Xe $5 p_{z}$ orbitals and constant $C$ to be fitted to the equilibrium value. For $\bar{d}_{i}=1.288 \AA, \quad S \simeq 0.055, C=3.75 \times 10^{-9}$ esu, and $\partial \mu / \partial \zeta=0.835 D /$ bohr.

At a preset electrode separation, the atom transfer can be viewed as the crossing of the barrier, which is momentarily reduced by $V_{P}$. In the asymmetric potential $U\left(\zeta, z ; V_{P}\right)$ the Xe atom can have certain quantum states $\Psi_{n}\left(\zeta, z ; V_{P}\right)$ with energies $E_{n}\left(z, V_{P}\right)$ and frequency $\omega_{n}\left(z, V_{P}\right)$ $(n=0,1,2, \ldots)$, which are obtained from the numerical solution of the Schrödinger equation. Here the state $n=0$ denotes the ground state. The bound states are localized in one of the wells. States having energy above the barrier may have comparable weights on both sides. The Xe atom, which is initially trapped in one of the wells, becomes excited and is transferred to the other well (by tunneling and ballistic process) and is relaxed. In what follows different processes (or mechanisms) contributing to the transfer rate at a given $z$ and $V_{P}$ are investigated.

\section{B. Thermally assisted transfer of Xe}

As substantiated by the experiment, ${ }^{5}$ the $\mathrm{Xe}$ atom can move spontaneously from one electrode, where it is initially bound to the other one by tunneling even for $V_{P}=0$. Of course, for $\mathrm{Xe}$ of mass $M$ the transmission probability $\mathcal{T}$ across an energy barrier of significant height and width is negligible. However, by decreasing the electrode separation $z$ the height of the energy barrier for adsorbed $\mathrm{Xe}$ is decreased. This may result in a significant transfer rate. The tunneling barrier for the $\mathrm{Xe}$ atom at the $i$ th quantum state localized on one of the electrodes is $\phi_{i}=Q-E_{i}\left(z, V_{P}\right)$. The thermal probability of Xe to be at the $i$ th state at temperature $T$ is $P^{i}(T)=e^{-E_{i} / k_{B} T / \Sigma_{j} e^{-E_{j} / k_{B} T}}$ where the summation in$\operatorname{dex} j$ runs over all localized states on the same site of Xe. The transmission probability $\mathcal{T}_{i}$ for the state $\Psi_{i}$ is less than unity for $\phi_{i}>0$, but becomes unity if $\phi_{i} \leqslant 0$ or $E_{i}$ lies above the barrier. The latter corresponds to the ballistic transfer of Xe.

As seen in Fig. 4(a), the applied pulse voltage $V_{P}$ induces asymmetry in the potential energy, which is otherwise symmetric for the system at hand. The eigenstates for the Xe atom are modified; while the well near the (left) electrode where the Xe is initially bound has fewer states, the (right) well at the other side (where the positive bias is applied) becomes deeper and has more eigenstates. Normally, the lowest eigenstate of the right electrode is lower than the lowest state of the left electrode. Consequently, an atom that is in thermal equilibrium at the left side is in a nonequilibrium state after it is transferred to the right side, but it is equilibrated quickly by the dissipation of its energy. Energy of Xe is dissipated by the excitation of the electron-hole pair, and mainly by the creation of metal phonons in the electrode. Using the elastic continuum model, we estimate the lifetime of the first excited state $\tau_{r} \simeq 20$ ps for $z=9.6 \AA$ and $V_{P}=0.1 \mathrm{~V}$ (see also Refs. 16 and 35) and treat higher states, $\Psi_{n>2}$, within the harmonic approximation. As a result the lifetimes of the higher-lying states decrease with $1 / n$. The lifetime of an excited state of a few quantum numbers higher than the ground state becomes less than one period of Xe. Accordingly, Xe loses its energy just after the first "collision" with the right electrode. The net thermally assisted rate $\tau_{T B}^{-1}$ for tunneling and ballistic transmission is given by

$$
\tau_{T B}^{-1}=\sum_{i}\left[f_{i}^{l} \omega_{i} \mathcal{T}_{i}^{l} P_{i}^{l}(T)-f_{i}^{r} \omega_{i} \mathcal{T}_{i}^{r} P_{i}^{r}(T)\right] .
$$

Here, the first and second terms represent the right-going and left-going rates, respectively. Each term is multiplied by the probability of state $f_{i}$ to be at the related electrode. $P_{i}^{l}(T)$ is the thermal probability of the state $i$ relative to the lowest state of the left electrode, and $P_{i}^{r}(T)$ is the thermal probability relative to the lowest state of the right electrode. In Eq. (5) the contribution of the second term is neglected and hence a transfer rate only towards a positively biased electrode is considered in the tunneling process. However, $\mathcal{T}_{i}^{r}=1$ for the ballistic transfer (corresponding to $\phi_{i} \geqslant 0$ ) and hence the second term in Eq. (5) is not negligible. In Fig. 5 we show the thermally assisted transfer rates (tunneling and ballistic) calculated at $T=4 \mathrm{~K}$ for different values of $z$. We note the following features in this figure. First, the calculated transfer rates $\tau_{T B}^{-1}\left(z ; V_{P}\right)$ do not exhibit a power-law dependence. Also, $\tau_{T B}^{-1}\left(z ; V_{P} \rightarrow 0\right)$ is finite for unidirectional transfer. For $V_{P}=0, \tau_{T B}^{-1} \sim 10^{-10} \mathrm{sec}^{-1}$ at $z=9.8 \AA$, but one transfer may occur in each second at $z \leqslant 9.5 \AA$. This implies 

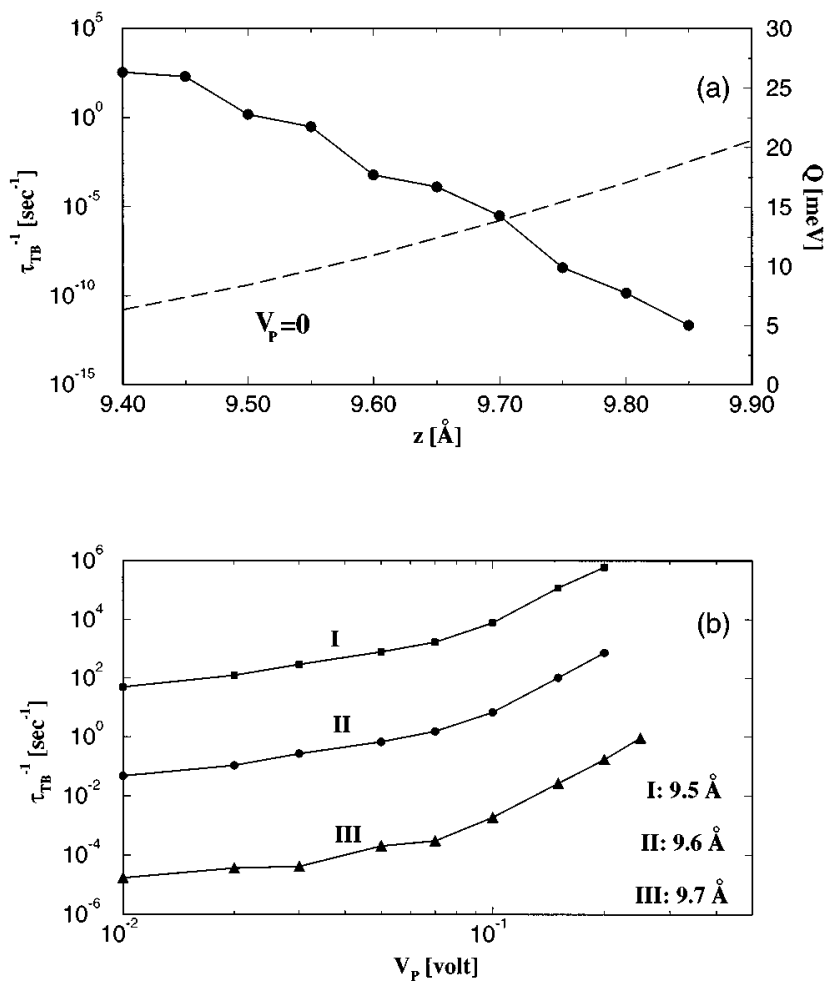

FIG. 5. (a) Rate of thermally assisted (ballistic and tunneling) transfer of $\mathrm{Xe}\left(\tau_{T B}^{-1}\right)$ and potential barriers vs the separation between $\mathrm{Pt}$ electrodes, $z$. (b) Same rate vs $V_{P}$ for $z=9.5 \AA$ (I), $z=9.6 \AA$ (II), and $z=9.7 \AA$ (III).

that the form of $U\left(\zeta, z ; V_{P}\right)$ is extremely important for realistic calculation of the rate of transfer. A potential energy function yielding a sharp barrier may be relatively less sensitive to the variation of $z$. Second, the variation of the transfer rates with the pulse voltage and especially with the separation $z$ is not smooth. The reason is that there are small numbers of discrete energy states involved in the transfer process. The last energy state below the energy barrier and the first state above the barrier make a dominant contribution to $\tau_{T B}^{-1}$. Modification of potential energy and hence lowering of the barrier with $V_{P}$ leads to discontinuous changes in the spectrum relative to the energy barrier. This may give rise to discontinuous changes in $\tau_{T B}^{-1}$.

\section{Momentum transfer by tunneling electrons}

Electrons that tunnel under the applied pulse voltage will be inelastically scattered by the adsorbed Xe atom. In accordance with the ballistic model of the electromigration of Fiks and Huntington, ${ }^{36}$ tunneling electrons transfer momentum $\Delta p$ to Xe during the collision process, which in turn induces so-called electron wind force acting on Xe. In this simple picture the energy transfer can be estimated by (I/e) $\times(\Delta p)^{2} / 2 M$. Walkup, Newns, and Avouris ${ }^{16}$ found that the electron wind force is one order of magnitude smaller than the dipole force. Following the approach of Ralls, Ralph, and Buhrman, ${ }^{37}$ Sáenz and Garcia ${ }^{15}$ expressed the temperature rise of Xe due to the energy transfer from the tunneling electrons by $\Delta T \sim C V_{P}^{2}$, where $C$ is taken as a constant to be determined from experimental data. The curve $\tau^{-1}\left(V_{P}\right)$ they calculated for a wide range of $C$ was quite different from the experimental data. It appears that the heating-assisted electromigration (or electron wind force) makes a small contribution to the net transfer rate.

\section{Excitations of Xe by inelastic electron tunneling}

A small fraction of electrons tunnel inelastically between two electrodes; they transfer energy to the Pt-Xe bond by two different processes: resonant tunneling and dipole excitation. Inelastic electron tunneling through an adsorbate has been treated earlier, ${ }^{38-40}$ and already proposed ${ }^{14,16,17}$ as a mechanism responsible for Xe transfer in the atom switch. In resonant tunneling, the tunneling electron is temporarily trapped in the Xe $6 s$ resonance. Owing to increasing excess charge on Xe the potential energy curve (or surface) in Fig. 4(a) is lifted to an excited energy state. In a time interval corresponding to the tunneling time $\tau_{t}$ the potential energy returns to its initial state, but Xe goes to a higher vibrational excited state, $\Psi_{n>0}$. This process, which is also referred to as the local polaron model, may involve even the Coulomb blockade, and may be quite complicated. A small fraction of tunneling electrons engages also in dipole excitation of the Xe-metal bond. This way the adsorbate may reach an excited vibrational state $\Psi_{n}$, for which $E_{n} \geqslant Q$, and be ready for transfer across (or over) the barrier. The excited adsorbate can reach this level in a single step (coherent process) created by one-electron tunneling or in multiple steps (incoherent process) created by subsequent tunneling of electrons. ${ }^{17}$ It is argued that $\tau_{r} \geqslant \tau_{t}$ makes the (incoherent) multiple vibrational excitation valid both in dipole excitation and the resonant tunneling process for Xe transfer. To calculate the contribution of both processes we use the formalism developed earlier ${ }^{39}$ and follow the below steps.

To calculate the contribution of resonant tunneling and dipole excitation processes one needs to know the fraction of electrons that engage in the inelastic tunneling. As proposed earlier ${ }^{16,39}$ the fraction of inelastic electrons contributing to dipole excitation from the ground state to the first excited state is approximately $\eta_{d} \simeq\left(\left\langle\psi_{1}|\zeta| \psi_{0}\right\rangle \partial \mu / \partial \zeta\right)^{2}\left(e a_{0}\right)^{-2}$, where $a_{0}$ is the Bohr radius. We estimate $\eta_{d}=2.75 \times 10^{-3}$ for the electrode separation $z=9.7 \AA$. Walkup, Newns, and Avouris ${ }^{16}$ estimated the fraction of electrons $\eta_{r}$ leading to the resonant tunneling process through the $6 s$ state, which lies $\sim 4 \mathrm{eV}$ above the Fermi level and has a width of $\sim 1 \mathrm{eV}$. They found $\eta_{r} \simeq 3 \times 10^{-4}$. Then the total fraction of inelastic tunneling is $\eta=\eta_{d}+\eta_{r}$ and the number of inelastic electron tunneling events per second is $\beta=(I / e) \eta$.

Based on the arguments above we adopt the (incoherent) single-step process, and within the harmonic approximation the rate of excitation (and also relaxation) from the $n-1$ state to the $n$ state, $g_{n-1}$ (or decay from $n$ to $n-1, r_{n}$ ) is larger than that from the ground state to the first excited state by a factor of $n$. Finally, the master equation for a singlestep process reads ${ }^{41}$

$$
\dot{\mathcal{P}}_{n}=r_{n+1} \mathcal{P}_{n+1}+g_{n-1} \mathcal{P}_{n-1}-\left(r_{n}+g_{n}\right) \mathcal{P}_{n}
$$

in terms of the occupation probability $\mathcal{P}_{n}$ of state $n, r_{n}$, and $g_{n}$. Since $g_{n-1}=\beta n$ and $r_{n}=\alpha n$ with $\alpha^{-1}=\tau_{r}$ in the harmonic approximation, the steady-state solutions become $\mathcal{P}_{n} \propto(\beta / \alpha)^{n}$. By disregarding the thermal distribution 


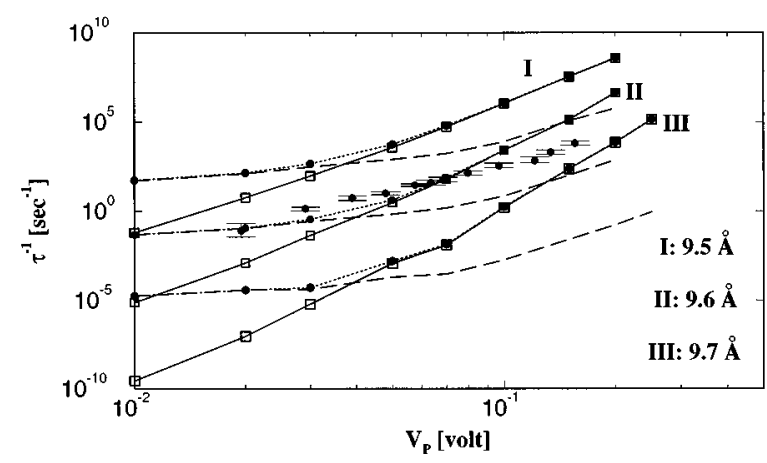

FIG. 6. Transfer rates of Xe vs pulse voltage $V_{P}$ are calculated for the electrode separation $z=9.5,9.6$, and $9.7 \AA$. The rate $\tau_{T B}^{-1}$ due to the thermally assisted tunneling and ballistic transfer and the rate $\tau_{I}^{-1}$ due to the inelastic tunneling are shown by dashed and continuous lines with squares. The total transfer rate of $\mathrm{Xe}$ is illustrated by the dotted curve. The error bars are the experimental data by Eigler, Lutz, and Rudge (Ref. 5) obtained from the rate of transfer of Xe between $\mathrm{Ni}$ and $\mathrm{W}$ electrodes.

(by taking $T=0$ ) we calculate the rate $\tau_{I}^{-1}$ of transfer due to the inelastic tunneling. Our results are shown by continuous lines with squares in Fig. 6. Note that this is the rate calculated by Walkup, Newns, and Avouris ${ }^{16}$ for the atom switch. As seen the rate $\tau_{I}^{-1}\left(V_{P} ; z\right)$ displays "approximately" a power-law dependence on the pulse voltage. For low $V_{P}$, the exponent of the power law is dependent on the electrode separation, but it converges to the same value for high $V_{P}$ for electrode separation $z \sim 9.6 \AA$. We also found that $\tau_{I}^{-1}$ is dependent on the value of the dipole moment and its variation.

\section{E. Total rate of transfer}

In order to calculate the total rate of Xe transfer we start with the thermal probabilities $P_{n}(T)$ of the $\mathrm{Xe}$ atom and consider that final steady-state probabilities $\mathcal{P}_{n}$ are modified by the tunneling current. The steady-state probability of occupation can be obtained from

$$
\mathcal{P}_{n}=\frac{P_{n}(T)}{F_{n}}+\sum_{j=0}^{n-1} P_{n j}^{D} .
$$

Here, $F_{n}=1+f c+f c^{2}+\cdots+f c^{L-n}, \quad f c=\beta / \alpha$, and $P_{n j}^{D}$ $=P_{j}(T) f c^{n-j} / F_{j}$. By taking $L$ sufficiently large, new probabilities are determined and are subsequently used in Eq. (5) to calculate the total rate $\tau^{-1}$ of transfer (including all contributions). Our results are illustrated in Fig. 6 by dotted curves for different values of $z$. In the same figure the contributions of thermally assisted tunneling and ballistic transfer are also shown. The experimental data by Eigler, Lutz, and Rudge 5 are included for the sake of comparison even if they belong to a different system of electrodes.

\section{CONCLUSIONS}

In this work the controlled lateral and perpendicular motions of $\mathrm{Xe}$ are investigated. The controlled lateral motion of $\mathrm{Xe}$ on the closed-packed (111) surface of Pt is induced by the $\mathrm{W}$ tip. The interaction between $\mathrm{Xe}$ and the $\mathrm{W}$ tip is described by empirical potentials and various modes of motion are revealed depending on the tip-surface separation. The corrugation of the potential energy of Xe is small owing to the closed-packed nature of the metal surface. Consequently, the Xe atom moves more freely as compared to the other surfaces and usually does not follow a straight trajectory in the surface plane. The effect of the tip becomes significant only for the tip separated less than $7.5 \AA$. Upon the interaction with the tip, Xe flops first sideways and is carried by the tip for $5 \AA<z<7 \AA$, whereas the adsorbed Xe atom is pushed by the tip at smaller separation. The range of $z$ leading to different modes in the controlled motion of Xe depends on the atomic arrangement of the apex of the tip and the tip material. These results are also relevant for boundary lubrication in tribology.

The controlled perpendicular motion of $\mathrm{Xe}$ is studied between two flat $\mathrm{Pt}(111)$ surfaces. The transfer of $\mathrm{Xe}$ is produced by the applied pulse voltage. The present model consisting of two flat electrodes is found suitable to analyze the contribution of various physical processes responsible for the controlled atom transfer since parameters such as tipstructure and tip-material affecting the transfer are not involved. We found that the form of the potential function $U(\zeta ; z)$, in particular the width of the barrier between two wells, is crucial in determining the contribution of the thermally assisted tunneling and ballistic transfer of Xe. Similarly, the value of the effective charge and its variation with the distance from the surface are important ingredients of the atom transfer. The contribution of electromigration (or electron wind force) in the transfer is known to be small. Our study reveals that the transfer due to thermally assisted tunneling and ballistic transfer of Xe between two $\mathrm{Pt}(111)$ electrodes is normally small but becomes dominant for small pulse voltage. Whereas the transfer rate due to the inelastic tunneling of electrons dominates the atom transfer in the range of normal and high pulse voltage. The calculated total rate does not yield a power-law dependence on the applied pulse voltage.

\section{ACKNOWLEDGMENTS}

We acknowledge stimulating discussions with Dr. A. Baratoff. This project was partially supported by the TUBITAK Grant No. TBAG-1085.
${ }^{1}$ D. M. Eigler and E. K. Schweizer, Nature 344, 524 (1990).

${ }^{2}$ L. J. Whitman, J. A. Stroscio, R. A. Dragoset, and R. J. Celotta, Science 251, 1206 (1991).

${ }^{3}$ I.-W. Lyo and P. Avouris, J. Chem. Phys. 93, 4479 (1990); Science 253, 173 (1991).
${ }^{4}$ H. J. Mamin, P. H. Guethner, and D. Rugar, Phys. Rev. Lett. 65, 2418 (1990)

${ }^{5}$ D. M. Eigler, C. P. Lutz, and W. E. Rudge, Nature 352, 600 (1991).

${ }^{6}$ Atomic and Nanometer-Scale Modification of Materials: Funda- 
mentals and Applications, edited by Phaedon Avouris (Kluwer Academic, Dordrecht, 1993).

${ }^{7}$ S. Ciraci and I. P. Batra, Phys. Rev. B 36, 6194 (1987); E. Tekman and S. Ciraci, ibid. 40, 10286 (1989); S. Ciraci, in Basic Concepts and Applications of Scanning Tunneling Microscopy and Related Techniques, edited by H. Rohrer, N. Garcia, and J. Behm (Kluwer, Amsterdam, 1989), p. 119.

${ }^{8}$ S. Ciraci, A. Baratoff, and I. P. Batra, Phys. Rev. B 41, 2763 (1990)

${ }^{9}$ R. Gomer, IBM J. Res. Dev. 30, 426 (1986).

${ }^{10}$ S. Ciraci, E. Tekman, A. Baratoff, and I. P. Batra, Phys. Rev. B 46, 10411 (1992); S. Ciraci, in Theory of Tip-Sample Interactions in Scanning Tunneling Microscopy III, edited by R. Wiesendanger and H.-J. Güntherodt, Springer Series in Surface Science Vol. 29 (Springer, Berlin, 1993), p. 179.

${ }^{11}$ A. Baratoff, S. Ciraci, and E. Stoll (unpublished).

${ }^{12}$ J. R. Cerda, P. L. de Andres, F. Flores, and R. Pérez, Phys. Rev. B 45, 8721 (1992); X. Bouju, C. Joachim, C. Girard and P. Sautet, ibid. 47, 7454 (1993).

${ }^{13}$ A. Buldum, S. Ciraci, and Ş. Erkoç, in Forces in Scanning Probe Methods, edited by H. J. Güntherodt, D. Anselmetti, and E. Meyer (Kluwer, Dordrecht, 1995), p. 149; J. Phys. Condens. Matter 7, 8487 (1995).

${ }^{14}$ S. Gao, M. Persson, and B. I. Lundqvist, Solid State Commun. 84, 271 (1992); J. Electron. Spectrosc. Relat. Phenom. 64/65, 665 (1993).

${ }^{15}$ J. J. Sáenz and N. Garcia, Phys. Rev. B 47, 7537 (1993).

${ }^{16}$ R. E. Walkup, D. M. Newns, and P. Avouris, Phys. Rev. B 48, 1858 (1993).

${ }^{17}$ G. P. Salam, M. Persson, and R. E. Palmer, Phys. Rev. B 49, 10655 (1994).

${ }^{18}$ M. Brandbyge and P. Hedegård, Phys. Rev. Lett. 72, 2919 (1994).

${ }^{19}$ J. A. Barker and C. T. Rettner, J. Chem. Phys. 97, 5844 (1992).

${ }^{20}$ J. E. Müller, Phys. Rev. Lett. 65, 3021 (1990).

${ }^{21}$ N. D. Lang, Phys. Rev. Lett. 46, 342 (1981).

${ }^{22}$ S. Ishi and B. Viswanathan, Thin Solid Films 201, 373 (1991).
${ }^{23}$ R. Pérez, F. J. Garcia-Vidal, P. L. de Andrés, and F. Flores, Surf. Sci. 307-309, 704 (1994); J. R. Cerda, F. Flores, P. L. de Andreas, and P. M. Echenique, Nuovo Cimento 15D, 451 (1993).

${ }^{24}$ Y. C. Chen, J. E. Cunningham, and C. P. Flynn, Phys. Rev. B 30, 7317 (1984).

${ }^{25}$ J. Topping, Proc. R. Soc. London Ser. A 114, 67 (1927).

${ }^{26}$ D. M. Eigler, P. S. Weiss, E. K. Schweizer, and N. D. Lang, Phys. Rev. Lett. 66, 1189 (1991).

${ }^{27}$ B. Gumhalter and D. M. Newns, Phys. Lett. 57A, 423 (1976).

${ }^{28}$ K. Wandelt and B. Gumhalter, Surf. Sci. 140, 355 (1984).

${ }^{29}$ A. Buldum, M.S. thesis, Bilkent University, 1994.

${ }^{30}$ D. M. Newns, Phys. Rev. 178, 1123 (1969).

${ }^{31}$ P. W. Anderson, Phys. Rev. 124, 41 (1961).

${ }^{32}$ F. Herman and S. Skillman, Atomic Structure Calculations, (Prentice-Hall, Englewood Cliffs, NJ, 1963).

${ }^{33}$ G. Schönhense, Appl. Phys. A 41, 39 (1986).

${ }^{34}$ N. D. Lang and W. Kohn, Phys. Rev. B 7, 3541 (1973); calculations based on the nonlocal exchange-correlation approximation in S. Ossicini and C. M. Bertoni, Europhys. Lett. 1, 661 (1986) yield relatively smaller values for $d_{i}$.

${ }^{35}$ B. N. J. Persson and R. Ryberg, Phys. Rev. B 32, 3586 (1985); J. A. Leiro and M. Persson, Surf. Sci. 207, 473 (1989).

${ }^{36}$ H. B. Huntington and A. R. Grone, J. Phys. Chem. Solids 20, 76 (1961); V. B. Fiks, Fiz. Tverd. Tela (Leningrad) 1, 16 (1959) [Sov. Phys. Solid State 1, 12 (1959)].

${ }^{37}$ K. S. Ralls, D. C. Ralph, and R. A. Buhrman, Phys. Rev. B 40, 11561 (1989).

${ }^{38}$ J. Kirtley, in Tunneling Spectroscopy, edited by P. K. Hansma (Plenum, New York, 1982); D. J. Scalapino and S. M. Marcus, Phys. Rev. Lett. 18, 459 (1967).

${ }^{39}$ B. N. J. Persson and J. E. Demuth, Solid State Commun. 57, 769 (1986); A. Baratoff and B. N. J. Persson, J. Vac. Sci. Technol. A 6, 331 (1988).

${ }^{40}$ J. W. Gadzuk, Phys. Rev. B 44, 13466 (1991).

${ }^{41}$ N. G. van Kampen, Stochastic Process in Chemistry and Physics, (North-Holland, Amsterdam, 1992). 